\title{
Solitons, solitonic vortices, and vortex rings in a cylindrical Bose-Einstein condensate
}

\author{
S. Komineas ${ }^{1}$ and N. Papanicolaou ${ }^{2}$ \\ ${ }^{1}$ Theory of Condensed Matter Group, Cavendish Laboratory, \\ Madingley Road, Cambridge CB3 OHE United Kingdom \\ ${ }^{2}$ Department of Physics, University of Crete, and Research Center of Crete, Heraklion, Greece
}

(Dated: November 25, 2018)

\begin{abstract}
Quasi-one-dimensional solitons that may occur in an elongated Bose-Einstein condensate become unstable at high particle density. We study two basic modes of instability and the corresponding bifurcations to genuinely three-dimensional solitary waves such as axisymmetric vortex rings and non-axisymmetric solitonic vortices. We calculate the profiles of the above structures and examine their dependence on the velocity of propagation along a cylindrical trap. At sufficiently high velocity, both the vortex ring and the solitonic vortex transform into an axisymmetric soliton. We also calculate the energy-momentum dispersions and show that a Lieb-type mode appears in the excitation spectrum for all particle densities.
\end{abstract}

PACS numbers: 03.75.Lm, 05.30.Jp, 05.45.Yv

\section{INTRODUCTION}

The possible occurrence of solitons in a Bose-Einstein condensate (BEC) was theoretically predicted sometime ago, within a one-dimensional (1D) Gross-Pitaevskii model [1, 2], but experimental observation was delayed because of the absence of a physical realization of a strictly 1D Bose gas. Nonetheless, similar coherent structures were recently observed in BECs confined in traps of varying geometry [3, 4] by a method (phase imprinting) that was directly inspired by the analytical structure of 1D solitons. Yet it has become clear that quasi-1D solitons are susceptible to various instabilities within the three-dimensional (3D) environment of realistic traps.

Indeed, a stability analysis based on the linear Bogoliubov-de Gennes (BdG) equations [5] revealed that an axisymmetric soliton is stable only at sufficiently low particle number or high aspect ratio in an elongated trap. The primary mode of instability was shown to result from non-axisymmetric perturbations, in the sense that a purely imaginary eigenvalue first appears in the spectrum of the BdG equations with azimuthal angular momentum $m=1$. This "snake instability" was further analyzed in Ref. [6] and argued to be responsible for a possible decay of a soliton into vortex rings and/or vortices. In fact, based on the above theoretical work, non-stationary vortex rings were experimentally detected in a spherical trap [7]. On the other hand, Brand and Reinhardt [8, 9] suggested that the primitive mode associated with the snake instability is a stable structure that may be called solitonic vortex.

The emerging picture is sufficiently perplexing to deserve closer attention. In some recent work [10, 11] we have carried out a detailed calculation of axisymmetric solitons in a cylindrical trap, which were shown to become unstable at high particle density even if we restrict ourselves to radial $(m=0)$ perturbations. The soliton was then shown to bifurcate to an axisymmetric vortex ring with lower energy. Here, we complete this work by allowing azimuthal deformations of the soliton, in order to eventually account for the primary $(m=1)$ instability discussed in Ref. 5, 6]. We thus obtain a reasonably complete description of the two basic modes of instability of the soliton and the corresponding bifurcations to axisymmetric vortex rings and non-axisymmetric solitonic vortices.

The simplest theoretical picture is obtained in the ideal limit of an infinitely elongated cylindrical trap, because static solitary waves may then be treated on equal footing with those propagating at nonzero velocity. Hence, all calculations presented in the main text pertain to an infinite cylindrical trap. In Sec. II we formulate the problem and briefly describe the numerical methods employed. The three basic types of static solitary waves (solitons, solitonic vortices, and vortex rings) and their relative stability are discussed in Sec. III, whereas solitary waves moving along the cylindrical trap at constant velocity are calculated in Sec. IV. The main conclusions are summarized in Sec. V, and some important technical details are relegated to two appendices. In particular, the case of a finite axisymmetric trap is discussed in Appendix B.

\section{FORMULATION}

An axisymmetric harmonic trap is characterized by a transverse confinement frequency $\omega_{\perp}$ and a longitudinal frequency $\omega_{\|}$. We first consider the special limit of a cylindrical trap, with $\omega_{\|}=0$, a restriction that is relaxed only in Appendix B. In this limit, physical units are rationalized as in Ref. [11]. Thus time is measured in units of $1 / \omega_{\perp}$ and distance in units of the transverse oscillator length $a_{\perp}=\left(\hbar / M \omega_{\perp}\right)^{1 / 2}$ where $M$ is the mass of each atom. Complete specification of the system also requires as input the linear density $\nu$ which is equal to the average number of particles per unit length of the cylindrical trap. An important dimensionless parameter is then given by $\gamma=\nu a$ where $a$ is the scattering length. A field rescaled according to $\Psi \rightarrow \sqrt{\nu} \Psi / a_{\perp}$ satisfies the 
rationalized Gross-Pitaevskii equation

$$
i \frac{\partial \Psi}{\partial t}=-\frac{1}{2} \Delta \Psi+V_{\mathrm{tr}} \Psi+g|\Psi|^{2} \Psi
$$

where $V_{\mathrm{tr}}=\left(x^{2}+y^{2}\right) / 2=\rho^{2} / 2$ is the trapping potential and $g=4 \pi \gamma$ is a dimensionless coupling constant that is the only free parameter. The conserved energy functional associated with Eq. (1) is given by

$$
W=\frac{1}{2} \int\left(|\nabla \Psi|^{2}+2 V_{\mathrm{tr}}|\Psi|^{2}+g|\Psi|^{4}\right) d V
$$

and yields energy in units of $\gamma_{\perp}\left(\hbar \omega_{\perp}\right)$ where $\gamma_{\perp}=\nu a_{\perp}=$ $\gamma\left(a_{\perp} / a\right)$.

We have also considered various forms of dissipative dynamics, mainly as relaxation algorithms for the calculation of stationary states. The simplest possibility is to make the replacement $i \partial \Psi / \partial t \rightarrow(i-\zeta) \partial \Psi / \partial t$ in Eq. (11), where $\zeta$ is some dissipation constant. An extreme special case widely employed in numerical simulations [6] is the fully-dissipative dynamics obtained by the replacement $i \partial \Psi / \partial t \rightarrow-\partial \Psi / \partial t$. However, in either case, particle number conservation is violated and special precautions are necessary to enforce a definite number of particles.

An interesting alternative that preserves particle number was originally introduced by Carlson [12] for the study of the homogeneous BEC and is simply adapted to the case of a confined BEC. Actually, we have employed a special case of Carlson's dissipative dynamics governed by

$$
i \frac{\partial \Psi}{\partial t}-\zeta \Psi \frac{\partial}{\partial t}|\Psi|^{2}=-\frac{1}{2} \Delta \Psi+V_{\mathrm{tr}} \Psi+g|\Psi|^{2} \Psi
$$

An immediate consequence of Eq. (3) is the continuity equation

$$
\frac{\partial n}{\partial t}+\nabla \cdot \boldsymbol{J}=0
$$

irrespective of the specific value of the dissipation constant $\zeta$. Here, $n=|\Psi|^{2}$ is the local particle density and

$$
\boldsymbol{J}=\frac{1}{2 i}\left(\Psi^{*} \nabla \Psi-\nabla \Psi^{*} \Psi\right)=n \nabla \phi
$$

is the usual current density, where $\phi$ is the phase of the wave function $\Psi=\sqrt{n} e^{i \phi}$. Therefore, the total number of particles is indeed conserved by the evolution equation (3). However, the total energy defined from Eq. (2) dissipates according to

$$
\frac{d W}{d t}=-\zeta \int\left(\frac{\partial n}{\partial t}\right)^{2} d V
$$

as long as the particle density $n$ remains time dependent.

Although both types of dissipation discussed above are employed in our numerical calculations, Carlson's proposal has some advantages for our current purposes. We have thus developed a discrete version of Eq. (3) on a cubic lattice to test the stability of solitons and eventually obtain rough estimates of other possible stationary states. These estimates are then fed into a NewtonRaphson algorithm of the type considered in Ref. 11] to obtain our final results more accurately as well as efficiently. The latter algorithm is currently upgraded to a fully-3D iterative scheme that does not necessarily enforce axial symmetry. Explicit results are discussed in the following sections.

\section{STATIC SOLITARY WAVES}

We first consider a special class of stationary states obtained by making the substitution $\Psi(\boldsymbol{r}, t) \rightarrow e^{-i \mu t} \Psi(\boldsymbol{r})$ in Eq. (1) to derive the static equation

$$
\mu \Psi=-\frac{1}{2} \Delta \Psi+\frac{1}{2} \rho^{2} \Psi+g|\Psi|^{2} \Psi,
$$

where $\mu$ is the chemical potential (in units of $\hbar \omega_{\perp}$ ) required to enforce a definite number of atoms. The simplest and most important solution of Eq. (7) is the ground state wave function $\Psi=\Psi_{0}(\rho)$ which depends only on the radial distance $\rho=\left(x^{2}+y^{2}\right)^{1 / 2}$ and may be chosen to be real and positive. It also satisfies the constraint

$$
\int_{0}^{\infty} 2 \pi \rho d \rho \Psi_{0}^{2}(\rho)=1
$$

by a specific choice of the chemical potential $\mu=\mu(\gamma)$ for each value of the dimensionless coupling constant $\gamma$. Explicit illustrations of the ground state may be found in Ref. [11].

A more general class of excited self-consistent states is obtained by solving Eq. (7) with the same chemical potential, as is appropriate on an infinitely elongated trap, and boundary conditions

$$
\lim _{z \rightarrow \pm \infty}|\Psi(x, y, z)|=\left|\Psi_{0}(\rho)\right|, \quad \lim _{z \rightarrow \pm \infty} \frac{\partial \Psi}{\partial z}=0 .
$$

The class of solutions actually discussed in the present paper is further restricted by the parity relation

$$
\Psi(x, y,-z)=\Psi^{*}(x, y, z)
$$

modulo an overall constant phase that is set equal to zero. Relation (10) is obviously consistent with Eq. (7).

To conclude the general description, we also consider the excitation energy defined as

$$
E=W-W_{0}-\mu \int\left(|\Psi|^{2}-\left|\Psi_{0}\right|^{2}\right) d V
$$

where both $W$ and $W_{0}$ are calculated from the energy functional (2) applied for a general self-consistent state $\Psi$ and the ground state $\Psi_{0}$, respectively. The presence in Eq. (11) of a term that is proportional to the chemical potential $\mu$ ensures that we are calculating the energy 


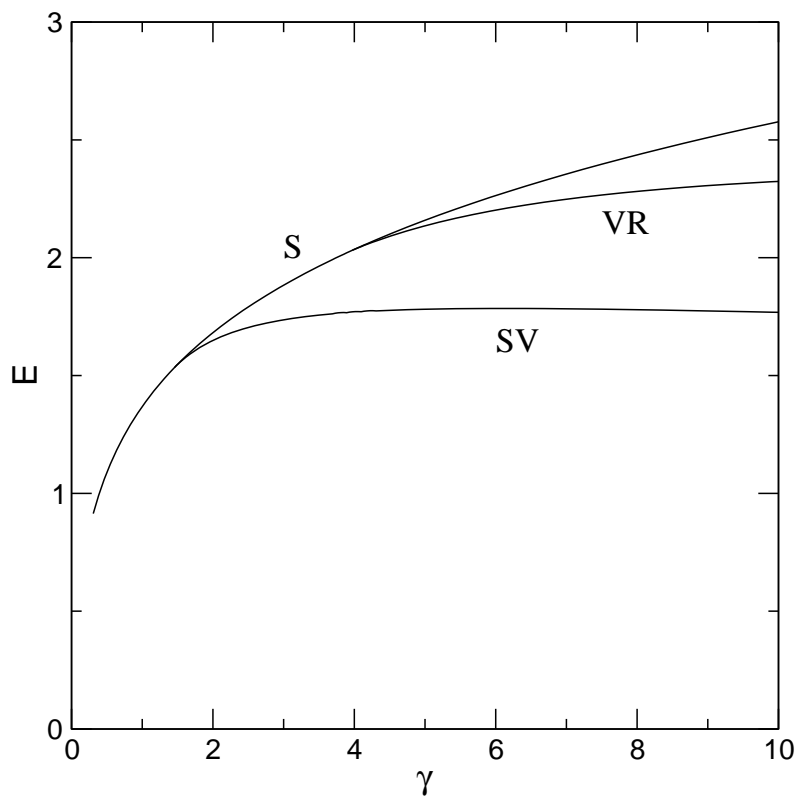

FIG. 1: Excitation energy $E$ in units of $\gamma_{\perp}\left(\hbar \omega_{\perp}\right)$ as a function of the dimensionless coupling constant $\gamma$, for static solitary waves such as a soliton (S), a solitonic vortex (SV), and a vortex ring (VR). Bifurcations occur at the two critical couplings $\gamma_{0}=1.5$ and $\gamma_{1}=4$.

difference between two states with the same number of particles.

In recent work 10, 11] we restricted ourselves to axisymmetric states described by a wave function of the form $\Psi=\Psi(\rho, z)$. The simplest possibility is a static (black) soliton whose wave function is odd under the parity reflection $z \rightarrow-z$, and purely imaginary in view of the phase convention adopted in Eq. (10). The excitation energy of a static soliton calculated from Eq. (11) is illustrated in Fig. 11 as a function of the dimensionless coupling constant $\gamma$.

When $\gamma$ exceeds the critical value $\gamma_{1}=4$, a new class of axisymmetric solitary waves emerges with the standard characteristics of vortex rings [10,11]. Unlike the case of a homogeneous BEC, a vortex ring may now be static and is described by a complex wave function that satisfies the parity relation $\Psi(\rho,-z)=\Psi^{*}(\rho, z)$. The calculated excitation energy of a static vortex ring is also shown in Fig. 1 and is seen to smoothly bifurcate from the soliton branch at the critical coupling $\gamma_{1}$, while it remains consistently lower than the soliton energy for $\gamma>\gamma_{1}$. Therefore, one may conclude that a radial $(m=0)$ perturbation of a soliton will render it unstable when $\gamma>\gamma_{1}$ and eventually transform it into an axisymmetric vortex ring.

One is tempted to presume that the soliton is stable for $\gamma<\gamma_{1}$. However, the linear stability analysis of Ref. 5, 6] indicates that the primary soliton instability sets in at a lower critical coupling $\gamma_{0}<\gamma_{1}$ through azimuthal $(m=1)$ perturbations. To probe for such a possibility, we solve the dissipative Eq. (3) with initial condition supplied by the calculated static soliton which is found to remain sta-

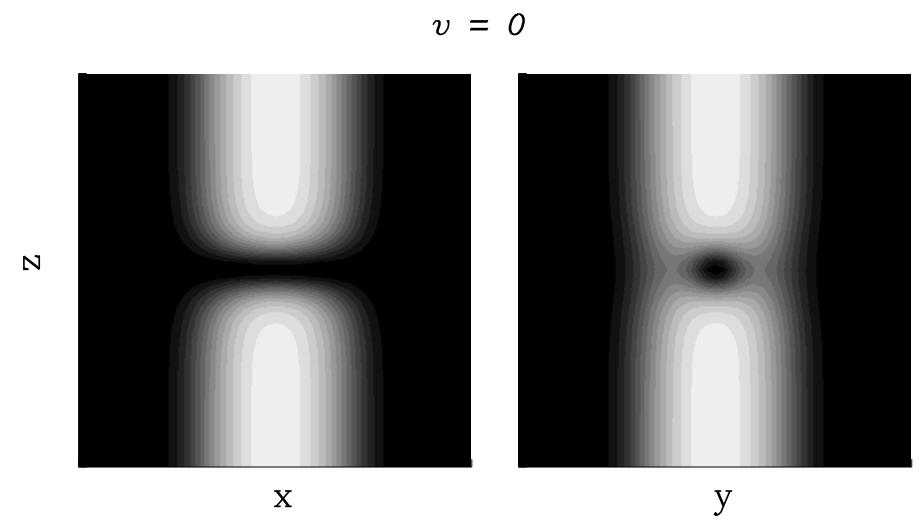

FIG. 2: Profile of a static $(v=0)$ solitonic vortex for $\gamma=$ 3 , illustrated through density plots over two planes that are perpendicular to each other and both contain the symmetry $(z)$ axis of the cylindrical trap. Regions with high particle density are bright while regions with zero density are black. The size of each frame is $[-5,5] \times[-5,5]$.

ble at all times when $\gamma<\gamma_{0}=1.5$. However, for $\gamma>\gamma_{0}$, the inherent numerical noise introduces an instability that transforms the soliton into a non-axisymmetric structure which is subsequently rectified by feeding it into a $3 \mathrm{D}$ Newton-Raphson iterative algorithm that is designed to directly solve the static equation (7).

We thus obtain a static solitary wave that is partially illustrated in Fig. 2 for $\gamma=3$ through density plots over two planes that are perpendicular to each other and both contain the $z$ axis. We have furthermore examined the phase to establish that the calculated wave function describes a deformed vortex that pierces through the trap in a direction that is perpendicular to its symmetry $(z)$ axis. In Fig. 2 the vortex axis is identified with the $x$ axis as a matter of convention, whereas a degenerate class of equivalent configurations may be obtained by simple azimuthal rotations around the $z$ axis. The resulting solitary wave displays the characteristics of a solitonic vortex calculated earlier by Brand and Reinhardt [8, 9] in traps of varying geometry.

The energy of the solitonic vortex is depicted in Fig. 1 as a function of $\gamma$ and is seen to emerge from the soliton branch at the critical coupling $\gamma_{0}=1.5$, while it remains smaller than the energy of both the soliton and the vortex ring for all $\gamma>\gamma_{0}$. The physical picture can be explained by returning to the initial-value problem associated with the dissipative Eq. (3) using the static soliton as initial condition. As mentioned already, the soliton remains stable for $\gamma<\gamma_{0}$ but decays into a solitonic vortex when $\gamma_{0}<\gamma<\gamma_{1}$. The pattern is more complex for $\gamma>\gamma_{1}$ where the soliton initially deforms radially to become an axisymmetric vortex ring which eventually decays into a stable solitonic vortex.

Therefore, a vortex ring is strictly speaking unstable. But our real-time simulations indicate that vortex rings are sufficiently long lived to be observed, as was actually the case in the experiment of Ref. 7] - albeit in the 
special limit of a spherical trap. On the other hand, it is curious that the predicted solitonic vortex has not yet been seen in experiment.

The picture is completed with a few remarks. One should expect that there exists a series of additional critical couplings (densities) at which the soliton bifurcates into multiple vortex rings and/or solitonic vortices. For example, a double vortex ring was calculated in Ref. 11] for $\gamma>\gamma_{2}=12$. In other words, we have only described the two basic patterns of instability that correspond to the appearance of unstable modes in the $m=0$ and $m=1$ sectors of the linear BdG equations. These two modes may be dominant at intermediate densities, but more complicated patterns should be expected to occur at higher densities, as is evident in the experiment of Ref. 7] which is further discussed in Appendix B.

\section{MOVING SOLITARY WAVES}

All three types of static solitary waves discussed in the preceding section are special members of a more general class of stationary states that propagate along the cylindrical trap with constant velocity $v$. These are obtained by making the substitution $\Psi(\boldsymbol{r}, t) \rightarrow e^{-i \mu t} \Psi(x, y, \xi)$ in Eq. (1), with $\xi=z-v t$, to derive the stationary differential equation

$$
\begin{aligned}
\mu \Psi-i v \frac{\partial \Psi}{\partial \xi} & =-\frac{1}{2} \Delta \Psi+\frac{1}{2} \rho^{2} \Psi+g|\Psi|^{2} \Psi \\
\Delta & =\frac{\partial^{2}}{\partial x^{2}}+\frac{\partial^{2}}{\partial y^{2}}+\frac{\partial^{2}}{\partial \xi^{2}}
\end{aligned}
$$

which reduces to the static equation (7) at $v=0$. The boundary conditions (9) and the parity relation (10) are also generalized by the simple replacement $z \rightarrow \xi$.

Equation (12) is solved by feeding a static $(v=0)$ solitary wave into the Newton-Raphson algorithm and by upgrading the velocity typically in steps of $\delta v= \pm 0.1$. The results for axisymmetric solitons and vortex rings already presented in Ref. 10, 11] have been confirmed using the currently employed fully-3D algorithm. Therefore, in the following, we shall mostly discuss the case of a moving solitonic vortex for which no calculation has been given in the literature. The static solitonic vortex of Fig. 2 is significantly reshaped when it moves, as is illustrated in Fig. 3 for two values of the velocity $(v=0.4$ and 0.8 ) that are both smaller than the speed of sound $c=1.3$ calculated for $\gamma=3[11$. The $v=0.4$ entry of Fig. 3 makes it clear that a moving solitonic vortex is shifted off center. While the vortex axis remains within the basal $x y$ plane, it is clearly displaced away from the center of the trap. The displacement increases with increasing speed until we reach a critical speed $v_{0}=0.8$ where the vortex disappears from the trap, in the sense that the solitary wave is converted into a gray axisymmetric soliton. This implies, in particular, that the density plots over the $x z$
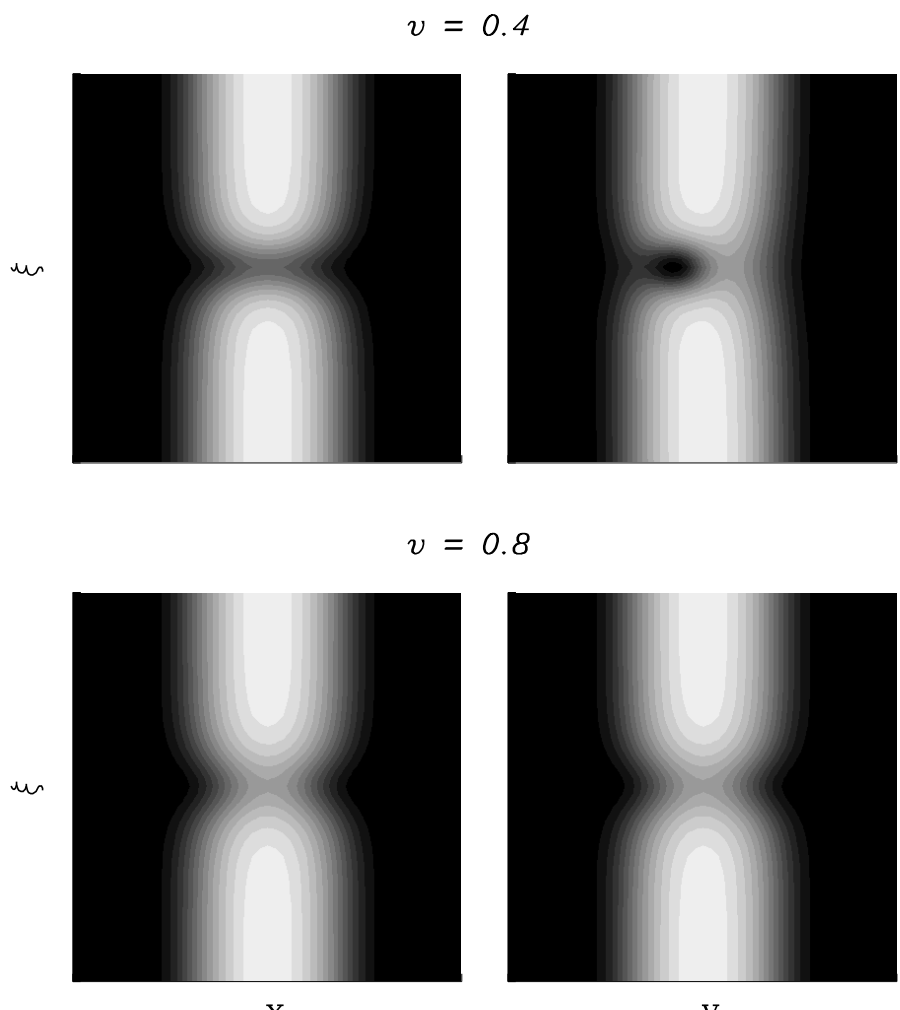

$v=0.8$

FIG. 3: Profile of a moving solitonic vortex for $\gamma=3$ and two values of the velocity $v=0.4$ and 0.8 . Conventions are the same as in Fig. 2] except that $\xi=z-v t$ now measures the distance from the center of the moving solitonic vortex. The speed of sound calculated at $\gamma=3$ is $c=1.3$.

and $y z$ planes become identical, as is apparent in the $v=0.8$ entry of Fig. 3

For $v_{0}<|v|<c$ the calculated solitary wave is indeed identical to a moving axisymmetric soliton and reduces to a weakly nonlinear soundlike pulse in the limit $|v| \rightarrow c$. A corollary of this discussion is that a high-speed soliton can be stable even for couplings $\gamma$ that are greater than the critical coupling $\gamma_{0}=1.5$, in apparent agreement with a similar conclusion reached in Ref. 13] that is now made more precise. Specifically, for $\gamma<\gamma_{0}$ a solitonic vortex does not exist and the soliton is stable over the entire range of velocities $0<|v|<c$, as expected. For $\gamma>\gamma_{0}$, the soliton is stable over the limited range $v_{0}<|v|<c$, where $v_{0}=v_{0}(\gamma)$ is the critical speed at which the solitonic vortex is converted into a soliton and $c=c(\gamma)$ is the calculated speed of sound [11]. Clearly, $v_{0}\left(\gamma=\gamma_{0}\right)=0$, while a numerical investigation of the ratio $v_{0}(\gamma) / c(\gamma)$ shows that it approaches unity with increasing $\gamma$. However, we are not in a position to ascertain whether or not there exists a finite (critical) $\gamma$ above which $v_{0}=c$ and, hence, the soliton is not stable for any velocity. In any case, the solitonic vortex is the fundamental mode as long as $\gamma>\gamma_{0}$.

Further insight into the nature of a moving solitonic vortex is obtained by considering the behavior of its 


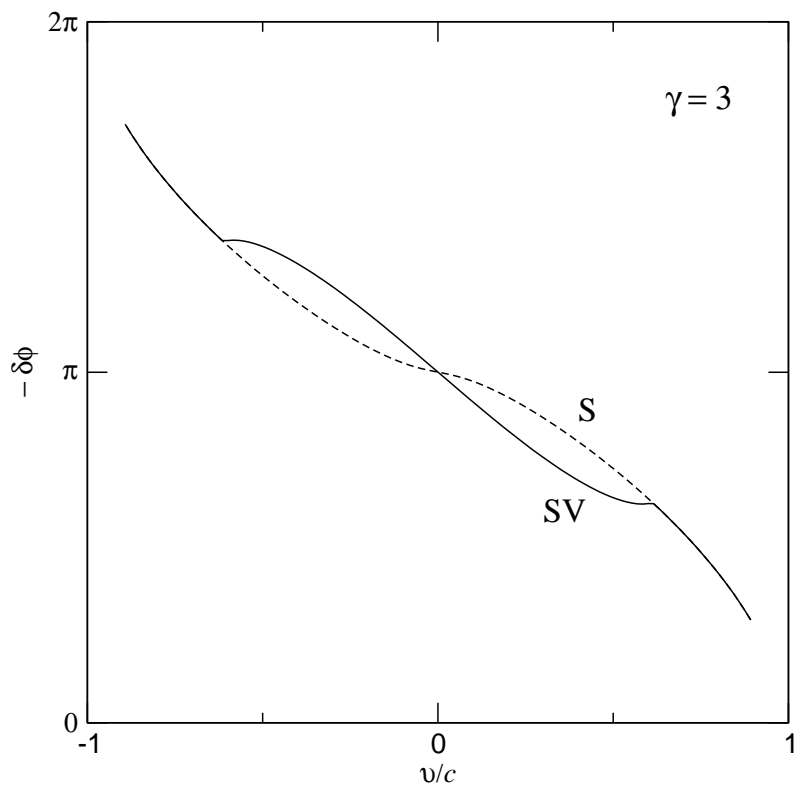

FIG. 4: Asymptotic phase difference as a function of velocity for a solitonic vortex (SV, solid line) and a soliton $(\mathrm{S}$, dashed line). The two curves merge at the critical velocities $v= \pm v_{0}$ where $v_{0}=0.6 c$.

phase $\phi=\phi(x, y, \xi)$ in the asymptotic limits $\xi \rightarrow \pm \infty$. Let $\phi_{ \pm}=\phi(x, y, \xi= \pm \infty)$ be the two asymptotic values of the phase, which are, in principle, some functions of $x$ and $y$. However, detailed numerical evidence suggests that $\phi_{+}$and $\phi_{-}$are, in fact, constants that depend only on the velocity $v$. This conclusion may be substantiated in part by an analytical argument briefly described in Appendix A. Therefore, the asymptotic phase difference

$$
\delta \phi=\phi_{+}-\phi_{-}
$$

depends only on the velocity $v$ and characterizes a solitary wave. Incidentally, the above conclusion applies to all three types of solitary waves discussed in the present paper and is pertinent to their experimental production through phase imprinting [3, 4].

As an illustration, we return to the solitonic vortex calculated for $\gamma=3$ and plot $-\delta \phi$ as a function of velocity by a solid line in Fig. [4 whereas the corresponding result for an axisymmetric soliton at $\gamma=3$ is shown by a dashed line. In both cases, $-\delta \phi=\pi$ for $v=0$, and $-\delta \phi=0(\bmod$ $2 \pi$ ) in the extreme limits $v \rightarrow \pm c$ which are not actually reached in Fig. 4 due to numerical inaccuracies. The same figure provides clear evidence for the conversion of a solitonic vortex into an axisymmetric soliton at the critical speed $v_{0}=0.8$ or $v_{0} / c=0.6$.

The preceding discussion of the asymptotic phase is also pertinent to the definition of the impulse of a solitary wave 11] which is now simplified to

$$
Q=\mathcal{P}-\delta \phi, \quad \mathcal{P}=\int n \frac{\partial \phi}{\partial z} d V
$$

where $\mathcal{P}$ is the usual linear momentum and $\delta \phi$ is given

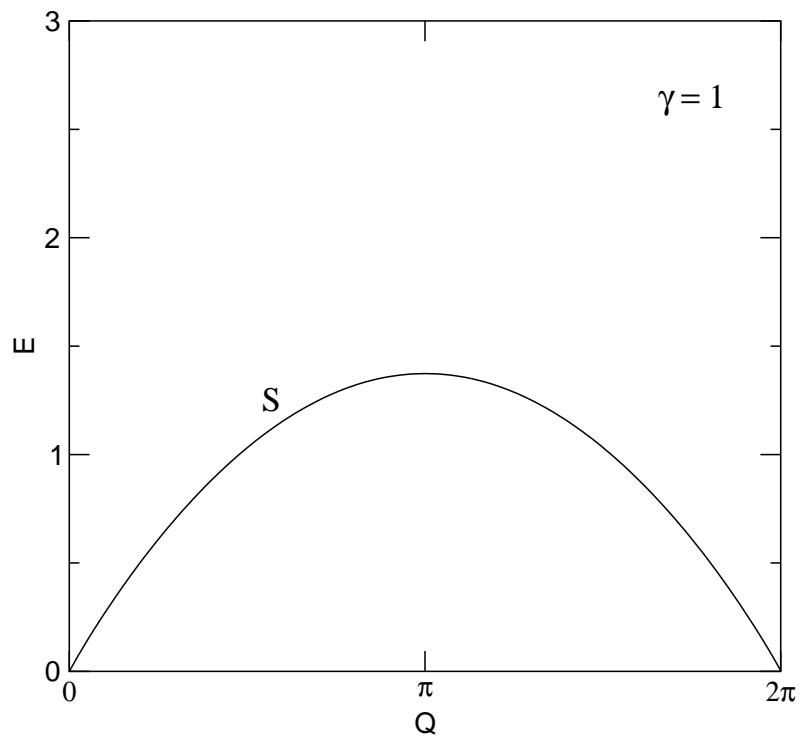

FIG. 5: Excitation energy $E$ in units of $\gamma_{\perp}\left(\hbar \omega_{\perp}\right)$ vs impulse $Q$ in units of $\gamma_{\perp}\left(\hbar / a_{\perp}\right)$ for a soliton (S) at $\gamma=1$.

by Eq. (13). The impulse $Q$ is measured in units of $\hbar \nu=$ $\gamma_{\perp}\left(\hbar / a_{\perp}\right)$.

A solitary wave may then be characterized by the impulse $Q$ and the excitation energy $E$ defined in Eq. (11). Both $E$ and $Q$ are definite functions of the velocity $v$ that can be eliminated to yield the dispersion $E=E(Q)$. A nontrivial check of consistency is provided by the group velocity relation $v=d E / d Q$ which was accurately confirmed by our numerical calculation. For comparison purposes, we reproduce in Fig. 囵 the dispersion of a stable axisymmetric soliton calculated for $\gamma=1<\gamma_{0}$ in Ref. [11]. This dispersion displays the characteristics of a mode originally derived by Lieb [14] within a full quantum treatment of a homogeneous 1D Bose gas based on the Bethe Ansatz, and was later rederived by a semiclassical calculation based on the solitary wave of the 1D Gross-Pitaevskii model [15, 16]. A similar mode was also derived within an effective 1D model that is designed to approximately describe a cylindrical trap [17].

We are now in a position to calculate the dispersion of a moving solitonic vortex. The dispersion is shown by a solid line in Fig. [6] and is seen to retain the basic characteristics of a Lieb-type mode. We further plot by a dashed line the dispersion of an axisymmetric soliton which is expected to be unstable at $\gamma=3$. However, the two dispersions shown in Fig. [6] merge at two critical points that correspond to velocities $v= \pm v_{0}$ where $v_{0}=$ $0.6 c$ is the critical speed at which the solitonic vortex is converted into an axisymmetric soliton.

A subtle detail not seen in our limited illustrations is that the calculated soliton for $\gamma=3$ exhibits some ringlike features, when $v \neq 0$, which are not present for $v=0$ and also disappear before reaching the critical speed $v_{0}$. These features could be viewed as preliminary signals for the formation of fully-fledged vortex rings that occur for 


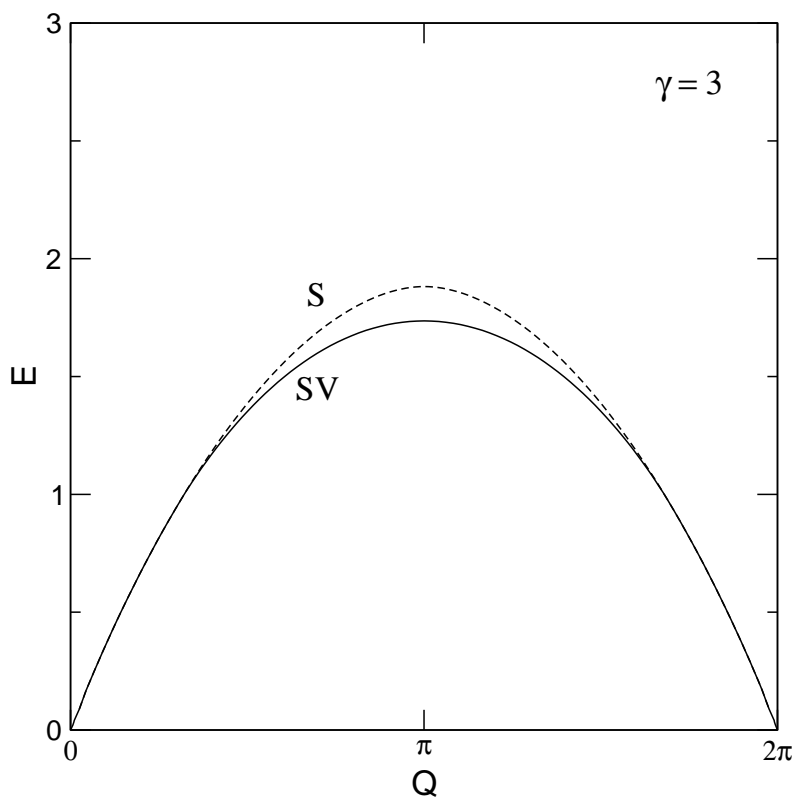

FIG. 6: Excitation energy $E$ vs impulse $Q$ for a solitonic vortex (SV, solid line) and a soliton (S, dashed line) at $\gamma=3$. The two dispersions merge at two points that correspond to velocities $v= \pm v_{0}$ where $v_{0}=0.6 c$.

stronger couplings in the region $\gamma>\gamma_{1}=4$.

Since a detailed calculation of both static and moving vortex rings was already described in Ref. [10, 11], we merely complete the picture by illustrating in Fig. 7 the dispersions of a soliton, a vortex ring, as well as a solitonic vortex calculated for $\gamma=7$ where all three possibilities occur according to the bifurcation pattern of Fig. [1] The solitonic vortex is still characterized by a Lieb-type dispersion that merges with the dispersion of the vortex ring at the critical speed $v_{0}=0.8 c$, where $c=1.6$ is the speed of sound calculated at $\gamma=7$. For $v_{0}<|v|<c$ both the solitonic vortex and the vortex ring become identical to a gray axisymmetric soliton. In fact, the vortex ring transforms into a soliton at a speed somewhat smaller than $v_{0}$.

Therefore, it is fair to say that the primary mode associated with the snake instability of a soliton is indeed the solitonic vortex [8] whose calculation has been significantly extended in the present paper to account for steady motion along a cylindrical trap. Actually, one may envisage a further generalization to a spinning solitonic vortex that undergoes rigid-body precession around the $z$ axis at constant frequency $\omega$, while it moves along the same axis at constant velocity $v$. One could then calculate the excitation energy $E=E(\omega, v)$, the impulse $Q=Q(\omega, v)$, and the azimuthal angular momentum $L_{z}=L_{z}(\omega, v)$ to eventually derive a dispersion of the form $E=E\left(Q, L_{z}\right)$. The Lieb-type dispersion of a solitonic vortex shown by a solid line in Figs. [6 and 7 would then be the lower edge of a continuum of states parametrized by the angular momentum $L_{z}$. This interesting possibility will not be discussed further in the

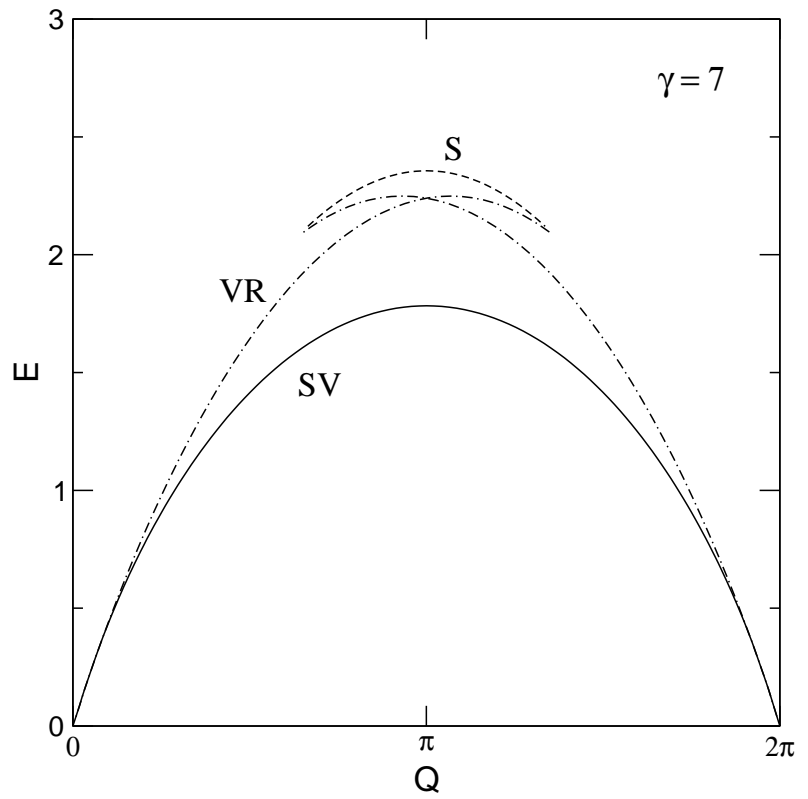

FIG. 7: Excitation energy $E$ vs impulse $Q$ for a solitonic vortex (SV, solid line), a vortex ring (VR, dotted dashed line) and a soliton (S, dashed line) at $\gamma=7$. The $\mathrm{SV}$ and $\mathrm{VR}$ dispersions merge at two points that correspond to velocities $v= \pm v_{0}$ where $v_{0}=0.8 c$.

present paper.

\section{CONCLUSION}

A phase imprint $\delta \phi$ applied between the two ends of an elongated trap leads to the formation of pure quasi-1D solitons only for weak couplings (densities) in the region $\gamma<\gamma_{0}=1.5$. For intermediate couplings in the region $\gamma_{0}<\gamma<\gamma_{1}=4$, solitons may be produced temporarily but they eventually decay into stable solitonic vortices. A new pattern emerges for $\gamma>\gamma_{1}$ where a phase imprint should initially produce unstable axisymmetric solitons that subsequently deform radially to become axisymmetric vortex rings and finally decay into solitonic vortices. This pattern is further complicated by the fact that high-speed vortex rings or solitonic vortices are indistinguishable from axisymmetric solitons.

It is thus important to reexamine the parameters of the experiment described in Ref. [3]. According to the estimates obtained in Appendix B, the length of the trap is significantly larger than its radius. Hence the approximation by an infinite cylindrical trap may be reasonable. Furthermore, a conservative estimate of the effective coupling leads to $\gamma \sim 7$ which lies in the region $\gamma>\gamma_{1}$. Therefore, all three types of solitary waves theoretically discussed in the present paper should have been produced in the original experiment of Ref. [3]. Also note that non-stationary vortex rings were actually detected in the experiment of Ref. 7] but on a spherical trap that is significantly different than the cylindrical trap considered 
here. Nevertheless, our present analysis can be extended to the case of a finite trap, as discussed in Appendix B.

Finally, the calculation of the present paper clearly demonstrates that a Lieb-type mode appears in the excitation spectrum for all densities, either in the form of a quasi-1D soliton (for $\gamma<\gamma_{0}$ ) or in the form of a solitonic vortex (for $\gamma>\gamma_{0}$ ). It is thus interesting to examine whether or not such a mode can be measured.

\section{Acknowledgments}

We are grateful to J. Brand for a stimulating discussion that brought the work of Ref. 8] to our attention, to N.R. Cooper for his constant interest in the present effort, and to A.S. Fokas, and N.S. Manton for a number of useful comments. This work was supported by the EPSRC under contract GR/R96026/01 (S.K.).

\section{APPENDIX A: ASYMPTOTIC PHASE}

The essence of the analytical argument given below was suggested to us by Fokas [18]. For a solitary wave traveling with constant velocity $v$, the continuity equation (4) may be written as $-v \partial n / \partial \xi+\nabla \cdot(n \nabla \phi)=0$. In the limits $\xi \rightarrow \pm \infty, n \rightarrow n_{0}(\rho)=\left|\Psi_{0}(\rho)\right|^{2}$ and all $\xi$ (or $z$ ) derivatives vanish on account of the boundary conditions (9). Hence, $\boldsymbol{\nabla}_{\perp} \cdot\left[n_{0}(\rho) \boldsymbol{\nabla}_{\perp} \phi_{ \pm}(x, y)\right]=0$, where $\boldsymbol{\nabla}_{\perp}$ is the $2 \mathrm{D}$ gradient operator and $\phi_{ \pm}(x, y)=\phi(x, y, \xi= \pm \infty)$ are the unknown asymptotic limits of the phase. If we further employ cylindrical coordinates $(x=\rho \cos \varphi, y=\rho \sin \varphi)$ the asymptotic form of the continuity equation reads

$$
\rho \frac{\partial}{\partial \rho}\left[\rho n_{0}(\rho) \frac{\partial \phi_{ \pm}}{\partial \rho}\right]+n_{0}(\rho) \frac{\partial^{2} \phi_{ \pm}}{\partial \varphi^{2}}=0 .
$$

For axisymmetric solitons and vortex rings $\phi_{ \pm}=\phi_{ \pm}(\rho)$ and thus the second term in Eq. (A1) vanishes. A first integral is then given by $d \phi_{ \pm} / d \rho=c_{1} / \rho n_{0}(\rho)$ where $c_{1}$ is some integration constant. Also note that the energy functional (2) contains a term $n(\partial \phi / \partial \rho)^{2}$ that becomes $n_{0}(\rho)\left(d \phi_{ \pm} / d \rho\right)^{2}=c_{1}^{2} / \rho^{2} n_{0}(\rho)$ at the two ends of the trap. Since the ground-state density $n_{0}(\rho)$ is finite at $\rho=0$ and vanishes in the limit $\rho \rightarrow \infty$, the above term would lead to a badly divergent integral over the radial distance $\rho$ unless the integration constant $c_{1}$ vanish. Hence, for an axisymmetric solitary wave with finite excitation energy, $d \phi_{ \pm} / d \rho=0$ and thus the asymptotic phases $\phi_{ \pm}$can depend only on the velocity $v$.

On the other hand, we have not been able to extend the above argument to the case of non-axisymmetric solitary waves for which both terms in Eq. A1 are potentially relevant. Nevertheless, our numerical results for the solitonic vortex discussed in the main text again suggest that the asymptotic phases $\phi_{ \pm}=\phi_{ \pm}(x, y)$ are, in fact, independent of $x$ and $y$.

\section{APPENDIX B: FINITE TRAPS}

A finite harmonic trap with axial symmetry is characterized by the two confinement frequencies $\omega_{\perp}$ and $\omega_{\|}$. We now introduce rationalized quantities that are in some respects different than those employed for the discussion of the infinite cylindrical trap. Time and distance are still measured in units of $1 / \omega_{\perp}$ and $a_{\perp}$ but the wave function is rescaled according to $\Psi \rightarrow \sqrt{N} \Psi / a_{\perp}^{3 / 2}$, where $N$ is the total number of atoms, and thus satisfies the constraint $\int|\Psi|^{2} d V=1$. The Gross-Pitaevskii equation is then formally identical to Eq. (11) but

$$
g=4 \pi \alpha, \quad V_{\mathrm{tr}}=\frac{1}{2}\left(\rho^{2}+\beta^{2} z^{2}\right)
$$

where $\alpha=N a / a_{\perp}$ and $\beta=\omega_{\|} / \omega_{\perp}$ are now the two independent dimensionless parameters. To make contact with the infinite trap, we calculate the average linear density from $\nu(z)=\left(N / a_{\perp}\right) \int|\Psi|^{2} d x d y$ and define the dimensionless quantity $\gamma(z)=a \nu(z)$ or

$$
\gamma(z)=\alpha \int|\Psi|^{2} d x d y
$$

which is the analog of the dimensionless coupling constant $\gamma$ employed in the main text, except that it now depends on $z$. Nevertheless, $\gamma(z)$ becomes increasingly uniform in the limit $\alpha \rightarrow \infty$ and $\beta \rightarrow 0$, holding $\gamma(0) \equiv \gamma$ fixed, which is the ideal limit of an infinite cylindrical trap studied in the main body of the paper.

As a simple illustration, we consider the Thomas-Fermi (TF) approximation of the ground state [19] to find that

$$
\gamma(z)=\frac{1}{16}\left(2 \mu-\beta^{2} z^{2}\right)^{2}
$$

where the chemical potential is given by $2 \mu=(15 \alpha \beta)^{2 / 5}$. Therefore, the estimated maximum value of $\gamma$ is given by $\gamma_{\max }=\gamma(0)=\mu^{2} / 4$. A more conservative estimate is the average value defined from $\gamma_{\mathrm{av}}=\alpha / L$ where $L=2 \sqrt{2 \mu} / \beta$ is the TF length of the trap and thus $\gamma_{\mathrm{av}}=(8 / 15) \gamma_{\max }$. The TF radius of the trap is accordingly given by $R=$ $\sqrt{2 \mu}$. For the parameters of the experiment described in Ref. 3] we find $R=2 \mu \mathrm{m}$ and $L=115 \mu \mathrm{m}$, while $\gamma_{\max }=$ 13 and $\gamma_{\mathrm{av}}=7$. These estimates are further discussed in the concluding section in the main text.

Now, static solitons, solitonic vortices, and vortex rings exist also in a finite trap and we have calculated them explicitly for various values of $\alpha$ and $\beta$ to confirm the general picture described in Sec. III. Here, we briefly summarize the basic facts. For each value of the aspect ratio $\beta$, there exist two critical couplings $\alpha_{0}=\alpha_{0}(\beta)$ and $\alpha_{1}=\alpha_{1}(\beta)$ that lead to a bifurcation pattern similar to that shown in Fig. 11 The critical coupling $\alpha_{0}$ coincides with the coupling where a purely imaginary eigenvalue first appears in the $m=1$ sector of the linear BdG equations 5]. Similarly, the critical coupling $\alpha_{1}$ is related to an instability that occurs in the $m=0$ sector, but the 


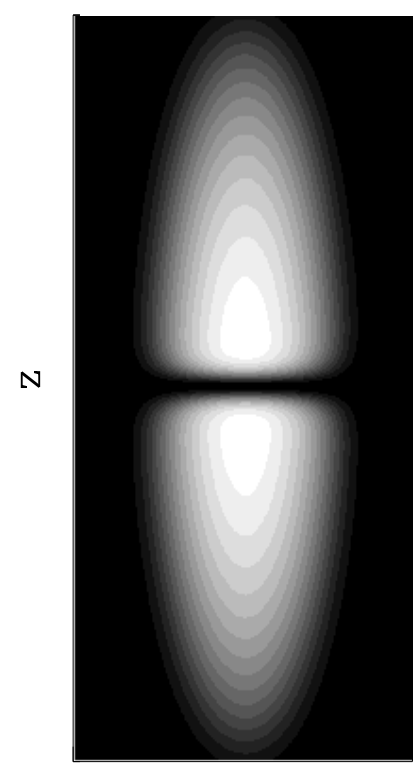

$\mathrm{X}$

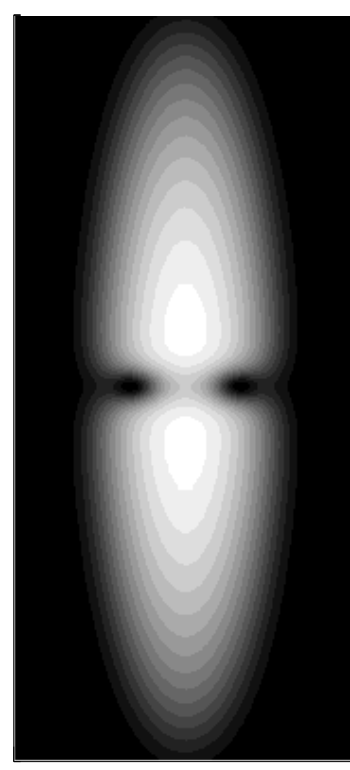

X
FIG. 8: Profiles of a static soliton (left panel) and a static vortex ring (right panel) on a finite trap with $\alpha=100$ and $\beta=1 / 4$, illustrated through density plots over a plane that contains the symmetry $(z)$ axis and cuts across the axisymmetric trap. The complete pictures can be obtained by simple revolution around the $z$ axis because both the soliton and the vortex ring are axisymmetric.

picture is now significantly complicated by the appearance of complex modes [6]. Our numerical solution of the BdG equations shows that there exists an intermediate critical coupling $\alpha_{1}^{\prime}<\alpha_{1}$ where two real eigenvalues with $m=0$ coalesce and eventually become complex for $\alpha>\alpha_{1}^{\prime}$. The precise behavior of the complex eigenvalues with increasing $\alpha$ depends on the specific value of $\beta$. A common feature is that complex eigenvalues as such again disappear before reaching the critical coupling $\alpha_{1}$. The latter is characterized by the fact that only one purely imaginary eigenvalue persists for $\alpha>\alpha_{1}$ and signals a new bifurcation that leads to the appearance of axisymmetric vortex rings.

As an example we consider the popular ratio $\beta=1 / 4$ for which we find $\alpha_{0}=10, \alpha_{1}^{\prime}=32, \alpha_{1}=39$. A static soliton exists for all $\alpha$ but is stable only for $\alpha<10$, while a static solitonic vortex appears as the most stable structure for $\alpha>10$. A new threshold occurs at $\alpha=39$ where a static vortex ring emerges with energy intermediate between that of a soliton and a solitonic vortex, in analogy with the situation on an infinitely cylindrical trap demonstrated in Fig. 11 Explicit illustrations of the currently calculated profiles are given in Figs. 89 for $\alpha=100$ and $\beta=1 / 4$ where all three types of static solitary waves are possible. For smaller $\beta$ the critical couplings $\alpha_{0}$ and $\alpha_{1}$ increase indefinitely. But the corresponding effective couplings $\gamma_{0}$ and $\gamma_{1}$, numerically calculated from Eq. (B2) applied for $z=0$, converge to the asymptotic values $\gamma_{0}=1.5$ and $\gamma_{1}=4$ that are in agreement with

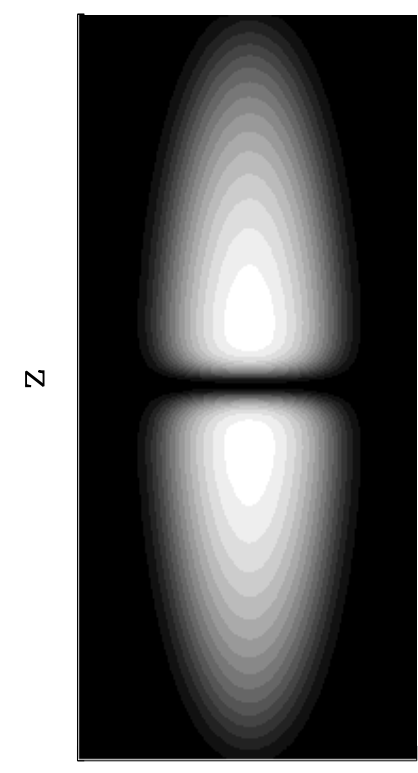

$\mathrm{X}$

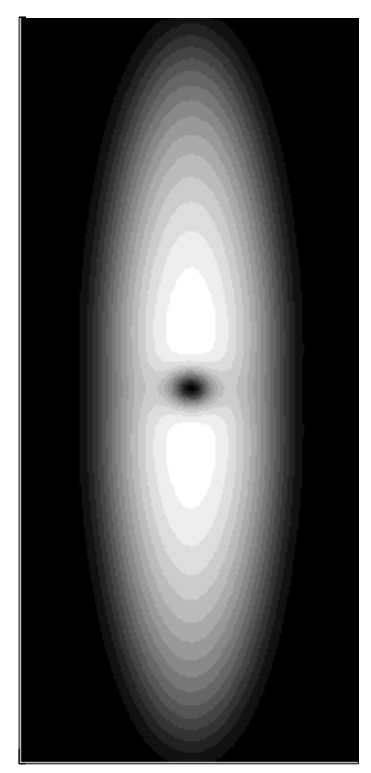

$\mathrm{y}$
FIG. 9: Profile of a static solitonic vortex on a finite trap with $\alpha=100$ and $\beta=1 / 4$, illustrated through density plots over two planes that are perpendicular to each other and both contain the symmetry $(z)$ axis. Note that the solitonic vortex is not axisymmetric.

the critical couplings obtained in the main text for an infinite cylindrical trap. It is also interesting to consider the special limit of a spherical $(\beta=1)$ trap in view of the experiment described in Ref. [7]. The critical coupling $\alpha_{0}$ vanishes at $\beta=1$ and thus the black soliton is never stable on a spherical trap. On the other hand, the solitonic vortex now becomes degenerate with the ordinary vortex and is stable for all $\alpha>0$. The vortex-ring threshold is here predicted to occur at $\alpha_{1}=5$. For the parameters of the actual experiment [7] we find $\alpha=430$ which is substantially larger than $\alpha_{1}$ and eventually explains the observation of multiple vortex rings.
[1] T. Tsuzuki, J. Low Temp. Phys. 4, 441 (1971).

[2] V.E. Zakharov and A.B. Shabat, Sov. Phys. JETP 37, 823 (1973).

[3] S. Burger, K. Bongs, S. Dettmer, W. Ertmer, K. Sengstock, A. Sanpera, G.V. Shlyapnikov, and M. Lewenstein,
Phys. Rev. Lett. 83, 5198 (1999).

[4] J. Denschlag, J. E. Simsarian, D. L. Feder, Charles W. Clark, L.A. Collins, J. Gubizolles, L. Deng, E.W. Hagley, K. Helmerson, W.P. Reinhardt, S.L. Rolston, B.I. Schneider, and W.D. Phillips, Science 287, 97 (2000). 
[5] A.E. Muryshev, H.B. van Linden van den Heuvell, and G.V. Shlyapnikov, Phys. Rev. A 60, R2665 (1999).

[6] D.L. Feder, M.S. Pindzola, L.A. Collins, B.I. Schneider, and C.W. Clark, Phys. Rev. A 62, 053606 (2000).

[7] B.P. Anderson, P.C. Haljan, C.A. Regal, D.L. Feder, L.A. Collins, C.W. Clark, and E.A. Cornell, Phys. Rev. Lett. 86, 2926 (2001).

[8] J. Brand and W.P. Reinhardt, J. Phys. B 34, L113 (2001).

[9] J. Brand and W.P. Reinhardt, Phys. Rev. A 65, 043612 (2002).

[10] S. Komineas and N. Papanicolaou, Phys. Rev. Lett. 89, 070402 (2002).

[11] S. Komineas and N. Papanicolaou, Phys. Rev. A 67, 023615 (2003).
[12] N.N. Carlson, Physica D 98, 183 (1996).

[13] A.E. Muryshev, G.V. Shlyapnikov, W. Ertmer, K. Sengstock, and M. Lewenstein, Phys. Rev. Lett. 89, 110401 (2002).

[14] E.H. Lieb, Phys. Rev. 130, 1616 (1963).

[15] P.P. Kulish, S.V. Manakov, and L.D. Faddeev, Theor. Math. Phys. 28, 615 (1976).

[16] M. Ishikawa and H. Takayama, J. Phys. Soc. Japan 49, 1242 (1980).

[17] A.D. Jackson and G.M. Kavoulakis, Phys. Rev. Lett. 89, 070403 (2002).

[18] A.S. Fokas, private communication.

[19] G. Baym and C.J. Pethick, Phys. Rev. Lett. 76, 6 (1996). 